Case Report

\title{
A Case Report of Avian Polyomavirus Infection in a Blue Fronted Parrot (Amazona aestiva) Associated with Anemia
}

\author{
Natalia Azevedo Philadelpho, Marta B. Guimarães, and Antonio J. Piantino Ferreira \\ Department of Pathology, School of Veterinary Medicine, University of São Paulo, Avenida Prof. Orlando Marques de Paiva 87, \\ 05508-900 São Paulo, SP, Brazil \\ Correspondence should be addressed to Antonio J. Piantino Ferreira; ajpferr@usp.br
}

Received 26 June 2015; Revised 29 September 2015; Accepted 5 October 2015

Academic Editor: Carlos Gutierrez

Copyright (c) 2015 Natalia Azevedo Philadelpho et al. This is an open access article distributed under the Creative Commons Attribution License, which permits unrestricted use, distribution, and reproduction in any medium, provided the original work is properly cited.

\begin{abstract}
An adult Blue Fronted Amazon parrot (A. aestiva) presenting with emesis, apathy, undigested seed in feces, and severe anemia was treated for approximately 2 months. Upon radiographic examination, an enlarged kidney was the only alteration. PCR for avian Bornavirus, Circovirus, and Polyomavirus was performed for the feces and blood. The results were positive for APV in both samples and negative for the other viruses. After 6 months, the feces from the same animal were negative for APV. Because the animal was positive for APV in both the feces and the blood, it is likely that these clinical symptoms were due to Polyomavirus infection. Severe anemia is an unusual clinical sign of Polyomavirus, and this study aims to identify novel differential diagnostic criteria for the disease.
\end{abstract}

\section{Introduction}

The Polyomavirus, one of the most important viruses in psittacines, is a highly infectious virus [1], reaching almost $100 \%$ infection rates in indoor aviaries. The disease has been described in North America, Europe, South Africa, Asia, and Australia [2-4] and is more common in budgerigars (Melopsittacus undulatus), lovebirds (Agapornis sp.), ringnecks (Psittacula krameri), conures (Aratinga spp., Nandayus nenday, and Pyrrhura spp.), and macaws (Ara spp.) $[4,5]$. However, the disease has also been described in passerines and Falconiformes [6, 7]. There are few reports in Amazon parrots worldwide and no description of APV in this species in Brazil. The aim of this report is to describe a possible clinical sign of APV in Amazon parrot.

\section{Case Presentation}

A Blue Fronted Amazon parrot (A. aestiva) aged 2 years and 4 months was presented at the Avian Clinic of the School of Veterinary Medicine and Animal Science of the University of São Paulo (FMVZ-USP) for emergency evaluation after three days of anorexia and apathy and one day of emesis. The bird was housed in a cage alone and was fed commercial pellet feed, sunflower seeds, fruits, and vegetables. On physical examination, the bird was in molt, with good pectoral muscle conformation, mild dehydration, and pale mucous membranes. Melena and polyuria were also observed. Treatment was initiated with enrofloxacin (IM $15 \mathrm{mg} / \mathrm{kg}$ Baytril 5\%, Bayer), iron $(20 \mathrm{mg} / \mathrm{kg}$ Ferrodex, Tortuga), vitamin B (3 mg/kg Vitamin B1, Labovet), and fluid (crystalloid solution $20 \mathrm{~mL} / \mathrm{kg}$, Equiplex).

The bird returned on day 3 , with no improvement in its clinical condition. Undigested seeds and fat in the feces were observed; however, there was no blood present. Because the animal was dehydrated and possibly anemic, no blood work was performed. Radiographic examination revealed an enlarged kidney and mildly dilated crop (Figure 1). On the same day, feces samples were collected for polymerase chain reaction (PCR) for avian Bornavirus, Circovirus, and Polyomavirus. Due to the presence of fat in the feces, oral pancreatin $(2 \mathrm{~g} / \mathrm{kg}$, compounded drug) and nystatin $(300,000 \mathrm{IU} / \mathrm{kg}$, Micostatin, Bristol) were prescribed.

There was no improvement on day 7 , and the animal was anemic, dehydrated, dyspneic, and still presenting with emesis, delayed emptying of the crop, and undigested seeds 


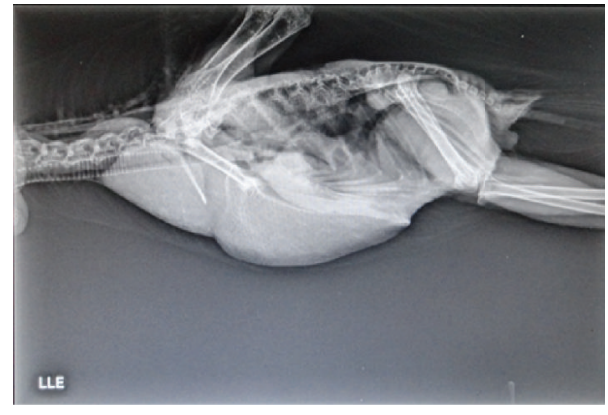

FIGURE 1: Radiographic image showing enlarged kidney and mildly dilated crop.

in the feces. For two days (days 8 and 9), the bird was unable to perch, staying at the bottom of the cage. The feces still had fat, but there was no blood. Because the animal was apathetic and anemic, a hematocrit test was performed, with a result of $33 \%$. Thus, we administered IM metoclopramide $(0.5 \mathrm{mg} / \mathrm{kg}$, Noprosil, Isofarma) and fluid (crystalloid solution $20 \mathrm{~mL} / \mathrm{kg}$, Equiplex, and colloidal solution $10 \mathrm{~mL} / \mathrm{kg}$, Voluven).

The following day (day 10), the animal had another emesis episode and was brought to the clinic. The bird received enrofloxacin, nystatin, and food through a gavage needle for three days. At day 14, the bird was slightly more active, and blood was collected for complete blood work and a drop of blood was sent for viral testing (Bornavirus, Circovirus, and Polyomavirus). Emesis and undigested seeds in the feces were still present. Fluid therapy with colloidal solution was again performed and the bird was given sucralfate SID (25 mg/kg, Sucrafilm, Sigma Pharma), thymomodulin, an immunostimulant that modulates maturation and function of T-lymphocytes and enhances the function of mature lymphocytes ( $2 \mathrm{mg} / \mathrm{kg}$, Leucogen, Aché), and vitamins. Hemogram results showed a severe nonregenerative, normocytic, normochromic anemia, with a hematocrit of $14 \%$ with discreet anisocytosis, polychromasia, and severe leucopenia, with no morphological alterations in the white blood cells. This anemia indicates decreased erythropoiesis. PCR results were positive for APV in the blood and feces and negative for Circovirus and $\mathrm{PaBV}$, confirming an active Polyomavirus infection.

On day 20, the bird started eating, was more active, and had no episodes of emesis, although it presented movements similar to emesis. Enrofloxacin treatment and nystatin treatment were suspended, but metoclopramide, sucralfate, immunostimulant, and vitamins were maintained. After seven days, the animal returned with a better appetite and was more active. Nystatin, vitamin, and immunostimulant were administered for another 10 days.

One month after the initial presentation, the bird started to vocalize and had a normal appetite. A hematocrit was performed with a result of $51 \%$, indicating the normalization of the hematological disorders. All medications were suspended, and the animal was discharged. After 6 months, the bird returned for a routine check-up (Figure 2). Feces were collected for PCR and were negative for APV.

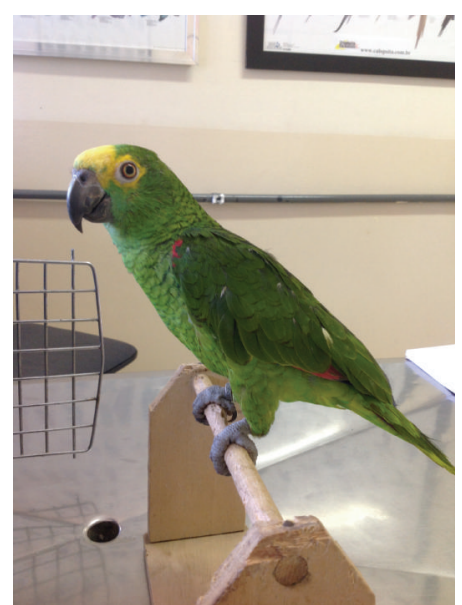

Figure 2: Blue Fronted Amazon parrot six months after the clinical presentation, with no clinical signs.

\section{Discussion}

The Polyomavirus is a highly infectious psittacine virus [1], reaching almost $100 \%$ infection rates in indoor aviaries. The disease has been described in North America, Europe, South Africa, Asia, and Australia [2,3] and is more common in budgerigars (Melopsittacus undulatus) [4], lovebirds (Agapornis sp.), ring-necks (Psittacula krameri), conures (Aratinga spp., Nandayus nenday, and Pyrrhura spp.), and macaws (Ara spp.) [5]. However, the disease has also been described in passerines [6] and Falconiformes [7].

There are few reports on Amazon parrots worldwide and no description of APV in this species in Brazil. Because clinical signs are not specific and are present in several differential diagnoses, investigation of Polyomavirus by the veterinarian is often neglected. However, there is also a reduced incidence of differential laboratory diagnosis of this disease compared with other viral infections in parrots. Thus, the prevalence of this disease in Brazil is unknown.

Clinical signs for APV are variable, depending on the species and the age. Death without any premonitory signs of the disease is reported in fledglings of various Psittaciformes [8], but abnormal feathers, skin discoloration, and abdominal distension are the most common clinical presentations [5, 9]. Other clinical signs include apathy, polyuria, diarrhea, dyspnea, weight loss, hemorrhage, and regurgitation $[1,4,5,10]$.

In the Amazon parrot, most infections are asymptomatic, and the few case reports are usually concomitant with Circovirus. As a result, few clinical signs of Polyomavirus are described in Amazon parrots, such as sudden death, depression, anorexia, weight loss, delayed crop empting, regurgitation, diarrhea, dehydration, subcutaneous hemorrhages, ataxia, and paralysis [8]. The possibility of chronic infections of psittacine birds with APV indicates the existence of an APV-positive subpopulation inside the population of captive psittacine birds that have subclinical infections that could serve as a viral reservoir [8].

All clinical signs presented in this paper are compatible with APV; however, severe resulting in a $14 \%$ hematocrit 
during viremia is a novel symptom compared to reports in the literature. The hematocrit observed was $14 \%$, with reduced hemoglobin and red blood cells, anisocytosis, and polychromasia, forming nonregenerative normocytic, normochromic anemia, indicating decreased erythropoiesis. Anemia can be caused by an acute or chronic kidney lesion due to lower production of erythropoietin, a hormone regulating the production of blood cells [11]. There are no case reports of avian Polyomavirus infection in the bone marrow, and unfortunately we did not perform a cytological evaluation of the bird bone marrow. However, a viral infection in the bone marrow cannot be discarded.

In this report, the animal had polyuria, clear signal kidney disease, and active infection by APV virus, commonly found in the renal parenchyma. Commonly, viral infections lead to anemia, a hemolytic process arising as a result of the body's immune response. Thus, one can consider the possibility of anemia caused by a nephropathy generated or exacerbated by viral infection. In psittacine birds with enlarged kidneys and other minor changes, glomerulopathy with a positive PAS (periodic acid-Schiff) reaction may indicate infection with avian Polyomavirus [12]. The PAS reaction in Polyomavirus occurs due to the, sometimes massive, deposition of immune complexes [12]. With the improvement of clinical signs and support care, anemia was resolved within a month after treatment.

Phalen et al. [1] reported the apparent thrombocytopenia in blood smears from a breeder with an APV infection. They indicated the possibility of this thrombocytopenia as the cause of hemorrhage resulting from the disease. The cause of the thrombocytopenia was not detected; however, these authors suggested the possibility of a viral thrombolysis or an induction of a disseminated vascular coagulation. Nevertheless, in this report, there was no change in the thrombocytes, but the blood count was performed only 14 days after the onset of viral presentation. Thus, there is the possibility of a normalization of this symptom because the bird showed early clinical improvement.

Although anemia is not commonly described, one should take into account that most of the birds die without clinical signs, and thus, it is not possible to perform additional tests such as blood work. Considering the commonly observed symptoms, such as hematuria and subcutaneous petechia [1], the possibility that anemia also occurs in other birds cannot be disregarded. It is known that, in non-psittacine species, general hemorrhage is described [10], which obviously can lead to mild anemia or even to severe anemia. In addition, infectious agent can cause nonregenerative, normocytic anemia. Birds tend to develop anemia due to the lack of erythropoiesis very fast and maybe due to the short erythrocyte half-life [11].

In addition to the severe anemia, another hematological alteration was observed. Severe leukopenia without alterations in the other white cells, which is common in acute viral infections, was an interesting finding. According to the hemogram, there were no obvious signs of a bacterial infection that could be acting in conjunction with APV.

Acute APV in adult Amazon parrots has been described and is frequently caused by immunosuppression, usually associated with concomitant Psittacine Beak and Feather Disease Virus (PBFDV) infection [4]. Considering this possibility, we also tested the feces and blood for both PBFDV and ABV (avian Bornavirus, an agent that normally causes emesis and undigested seeds in the feces) by PCR, and the bird was negative for both viruses. It is probable that another underlying cause could have led to immunosuppression and the appearance of the clinical symptoms, such as stress associated with changes in the weather, diet, or breeding.

The transmission may have occurred in several ways, even by contact with contaminated ectoparasites [13]. A major problem of this disease is the difficulty of diagnosis due to the nonspecific symptoms and transmission by asymptomatic animals. The virus is shed in the feces, skin desquamation, and fomites [14]. It is suggested that viremia precedes cloacal virus shedding, because viremia has always been detected prior to or concurrent with cloacal virus shedding. The duration between the onset of viremia and the onset of cloacal virus shedding appears to be only a few days in a typical infection [1]. Another factor that plays an important role in the spread of avian viral diseases is the lack of epizootic control of imported birds [3]. This clinical report documents a two-year-and-four-month-old A. aestiva, positive for APV in the feces and blood by PCR, presenting with emesis, undigested seeds in the feces, and severe anemia. Considering that the duration of the disease progression in this case, one month, corroborates with the literature [1,4] as well as with the positive PCR results in both the feces and the blood, there is strong evidence that the clinical signs were related to APV. This is important due to the small amount of reports of infection in this species and the severe anemia, a symptom rarely described in APV infections. To the authors' knowledge, this is the first report of APV described in the Blue Fronted parrot in the country, and thus, we aim to alert clinicians of birds to a new clinical sign to include in the differential diagnosis.

\section{Conflict of Interests}

The authors declare that there is no conflict of interests regarding the publication of this paper.

\section{Acknowledgment}

This work was supported by a grant received from Conselho Nacional de Desenvolvimento Científico e Tecnológico (CNPq Grant no. 453920/2014-4). Antonio J. Piantino Ferreira is a recipient of $\mathrm{CNPq}$ fellowships-1B.

\section{References}

[1] D. N. Phalen, C. S. Radabaugh, R. D. Dahlhausen, and D. K. Styles, "Viremia, virus shedding, and antibody response during natural avian polyomavirus infection in parrots," Journal of the American Veterinary Medical Association, vol. 217, no. 1, pp. 32$36,2000$.

[2] C.-M. Hsu, C.-Y. Ko, and H.-J. Tsai, "Detection and sequence analysis of avian polyomavirus and psittacine beak and feather 
disease virus from psittacine birds in Taiwan," Avian Diseases, vol. 50, no. 3, pp. 348-353, 2006.

[3] T. Piasecki and A. Wieliczko, "Detection of beak and feather disease virus and avian polyomavirus DNA in psittacine birds in Poland," Bulletin of the Veterinary Institute in Pulawy, vol. 54, no. 2, pp. 141-146, 2010.

[4] M. Szweda, A. KoŁodziejska, J. Szarek, and I. Babińska, "Avian polyomavirus infections in Amazon parrots," Medycyna Weterynaryjna, vol. 67, no. 3, pp. 147-150, 2011.

[5] D. N. Phalen, "Avian polyomavirus: my thoughts," American Federation of Aviculture Watchbird, vol. 10, pp. 28-39, 1998.

[6] G. Rossi, E. Taccini, and C. Tarantino, "Outbreak of avian polyomavirus infection with high mortality in recently captured Crimson's seedcrackers (Pyrenestes sanguineus)," Journal of Wildlife Diseases, vol. 41, no. 1, pp. 236-240, 2005.

[7] R. Johne and H. Müller, "Avian polyomavirus in wild birds: genome analysis of isolates from Falconiformes and Psittaciformes," Archives of Virology, vol. 143, no. 8, pp. 1501-1512, 1998.

[8] M. Rahaus and M. H. Wolff, "A survey to detect subclinical polyomavirus infections of captive psittacine birds in Germany," Veterinary Microbiology, vol. 105, no. 1, pp. 73-76, 2005.

[9] A. Ramis, K. S. Latimer, X. Gibert, and R. A. Campagnoli, "A concurrent outbreak of psittacine beak and feather disease virus, and avian polyomavirus infection in budgerigars (Melopsittacus undulatus)," Avian Pathology, vol. 27, no. 1, pp. 43-50, 1998.

[10] S. L. Lafferty, A. M. Fudge, R. E. Schmidt, G. V. Wilson, and D. N. Phalen, "Avian polyomavirus infection and disease in a green aracaris (Pteroglossus viridis)," Avian Diseases, vol. 43, no. 3, pp. 577-585, 1999.

[11] T. W. Campbell, "Hematology in birds," in Veterinary Hematological and Clinical Chemistry, M. Thrall, D. Barker, and T. Campbell, Eds., pp. 238-277, Lippincott Williams \& Wilkins, 2004.

[12] H. Gerlach, F. Enders, M. Casares, H. Müller, R. Johne, and T. Hänichen, "Membranous glomerulopathy as an indicator of avian polyomavirus infection in psittaciformes," Journal of Avian Medicine and Surgery, vol. 12, no. 4, pp. 248-254, 1998.

[13] J. Potti, G. Blanco, J. Á. Lemus, and D. Canal, "Infectious offspring: how birds acquire and transmit an avia polyomavirus in the wild," PLoS ONE, vol. 2, no. 12, Article ID e1276, 2007.

[14] B. Ritchie, Avian Viruses: Function and Control, Wingers Publishing, Lake Worth, Fla, USA, 1995. 

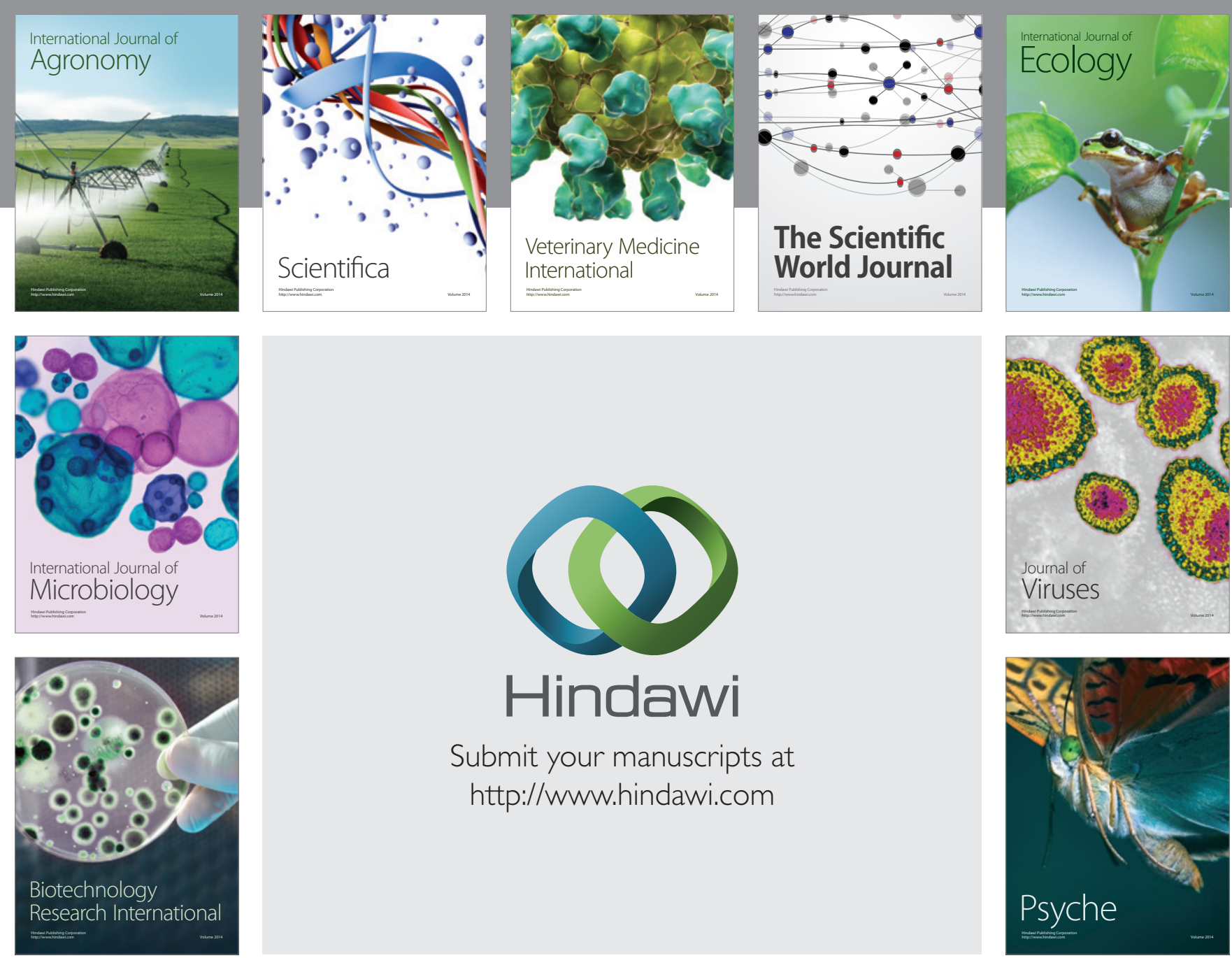

Submit your manuscripts at http://www.hindawi.com
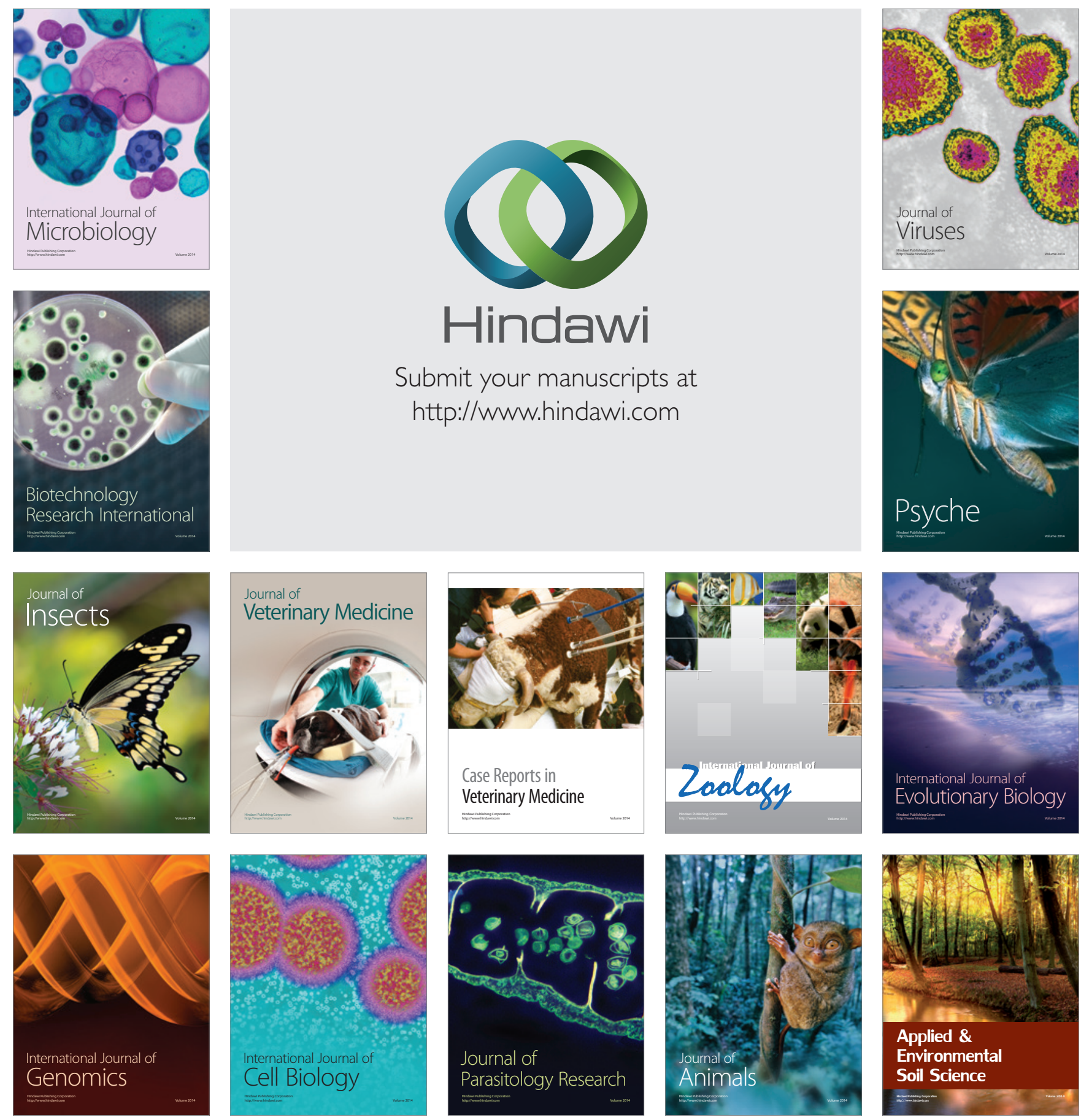